\title{
Editorial
}

\section{One Year into the COVID-19 Pandemic}

First and foremost, we would like to thank you all for your continued support to the International Academy for Clinical Hematology (IACH) and to its official peer-reviewed journal "Clinical Hematology International”. The year 2020 has been incredibly difficult for everyone. The pandemic might keep us in its grips for a little while longer, but the different vaccines are proving to be highly effective, and offer us hope for a return to the norm in the near future.

The COVID-19 pandemic sneaked suddenly into our lives. And then, a year like no other really began. A new era called "Zoom" was born and became part of everyone's household: Zoom conferences, Zoom family meetings, Zoom concerts, Zoom interviews, Zoom Pub Happy Hour, etc. In parallel, some non-evidence based COVID-19 'regulations' popped up here, there and everywhere. Examples of these were the requirement for you to sign a selfauthorization explaining why you, as a physician, need to leave the house and go to work in the hospital during the lock-down (French bureaucracy at its best), or the constant admonition for everybody to maintain a badly coined 'Social Distancing' of arbitrarily calculated, ever-changing square meter dimensions. In the meantime, overnight, everybody was transformed into experts on the interpretation of Polymerase Chain Reaction (PCR) results, the dangers of low arterial oxygen saturation levels, and the consequences of a cytokine storm. Fake news spread at light speeds. Some medical professionals, hitherto unknown to the lay public, suddenly became celebrities and household names, with their daily appearance at Press Conferences being eagerly awaited, not only to broadcast the latest contact-tracing results and change of policies, but also to allow the locked-down public to properly rate and compare their wardrobe, make-up and level of elegance.

Despite all this universal big mess, and although one always finds reasons to whinge and be mad at the upheaval brought about by COVID-19, 2020 was a very successful year for the IACH. During the first wave of the pandemic, the special COVID-19 series of weekly high-profile webinars featuring top experts in the field were extremely useful, and were able to reach a very large audience worldwide. Each webinar attracted more than a 1000 participants, who all praised the rich and diversified scientific content. The IACH annual meeting, and the world congress for controversies in multiple myeloma were rapidly converted into a virtual format freely accessible to all. These meetings included more than 10,000 participants from across the globe. The IACH monthly educational webinars continued to gain a huge popularity and tackled the hottest topics in the fields of malignant and non-malignant hematology. All IACH webinars remain freely accessible anytime to all, in order to reach the highest number of colleagues and stakeholders (http://www.clinical-hematology.org).

Moreover, the year 2020 ended up in a hopeful mood, with the introduction of the new "IACH journal club". The latter activity aims to provide a critical evaluation and appraisal of the rapidly growing scientific and medical literature. No physician or healthcare professional is able to 'digest' in a timely manner, the huge amount of knowledge being generated every day. Thus, there is a need to expand and balance each physician's scope of what is happening in the world of clinical research, while informing patients' care and research directions. With this background, the IACH launched a bi-monthly journal club (https://clinical-hematology.org/iach-journal-club/) to discuss recently published peerreviewed papers. The goal is to present first a summary of the chosen paper. Then, the discussion begins between the moderator and the panelists and audience. The final goal is to initiate more discussion about the topic, and how the results relate to the current knowledge and their impact on practice.

All the above activities allowed us at the IACH to keep the world of hematology connected when it mattered most, to remain active and enthusiastic, to quickly come to grips with the wealth of creative approaches that popped up at dizzying speeds. The dissemination of knowledge and advance of our field will always be at the forefront of everything we do. As we move forward, we will continue to develop new IACH activities and keep working to grow our worldwide network.

Now, as we edge closer toward the middle of 2021, we are feeling optimistic about the upcoming months. In this new issue of Clinical Hematology International, you will read some high-level clinical reviews and original reports. This issue marks the second anniversary of the journal. We thank all our readers, authors, reviewers and editors for their support, dedication, and investment. In this era of 'unprecedented' everything, we hope you will continue to enjoy the IACH activities. We honestly could not have done it without the amazing solidarity of the hematology community. 


\section{CONFLICTS OF INTEREST}

The authors declare they have no conflicts of interest.

Mohamad Mohty ${ }^{1, *},(\mathbb{D}$, Junia V. Melo

${ }^{1}$ Service d'Hématologie Clinique et Thérapie Cellulaire, Sorbonne University,

Saint Antoine Hospital and INSERM UMRs 938, Paris, France

${ }^{2}$ Faculty of Health and Medical Sciences, University of Adelaide, Adelaide, Australia

"Corresponding author.Email: mohamad.mohty@inserm.fr

Peer review under responsibility of the International Academy for Clinical Hematology

\section{AUTHORS' CONTRIBUTION}

Both authors contributed to writing and reviewing the manuscript. 